\title{
An Application of the Regression Technique to Generate Pseudo Relative Permeability Functions and Upscale Multiphase Flow Models
}

\author{
PALATNIK; Boris, Geomatic A/S, Norway \\ FØRLAND; Even, Statoil, Norway
}

Paper presented at the $5^{\text {th }}$ European Conference on the Mathematics of Oil Recovery, Leoben, Austria, 3-6 Sept.1996

\section{ABSTRACT}

The paper presents an application of a nonlinear regression based technique to estimate a set of pseudo relative permeability functions for a full-field numerical simulation model of oil and water flow during production from the Heidrun field on the Norwegian Continental Shelf.

A fine scale cross-section model was established with experimentally derived relative permeability curves. Pseudo relative permeability relationships were then generated by applying an iterative procedure on a coarse scale model resulting in a best match to the flow behaviour of the fine scale model. The fine scale simulation output functions of time for watercut in the producing well and for the pressure drop between the producer and the water injector were selected to be matched using a least squares objective function as a measure of overall deviation.

Two different techniques were applied to minimize the objective function. Both ensured stability of the successive estimates and smooth reduction of the objective function. Results for Heidrun are presented and discussed. 


\section{INTRODUCTION}

Pseudo relative permeability (PRP) functions have been widely used to account for small scale effects in fullfield numerical simulation models.

Relative permeability saturation functions based on laboratory measurements of cores (rock curves) are inappropriate for the macro sized cells of a full-field model. This is mainly due to small scale heterogeneity effects which are smoothed out on a larger scale, but also because of the invalid implications of numerical dispersion, and flow directionality.

Recent advances in reservoir characterisation, to a great extent related to implementation of stochastic modelling, have made it possible to generate high-resolution reservoir description models that contain millions of grid cells. Such models allow for representative pictures of real reservoir heterogeneity, integrating all available data and knowledge. What is probably most important, the advanced stochastic modelling packages provide consistent methods of scaling up reservoir parameters like porosity and permeability, obtained on high-resolution models, to full-field models. The scaling up of relative permeability functions (in fact, estimation of PRP curves) remains still a challenge.

The integration and averaging of fine grid data and results over the equivalent coarse grid cells, in order to derive pseudo characteristics, has been the subject of study for many years ${ }^{1-5}$. Several software packages for the purpose are currently in use. To the best of our knowledge none of the packages are universal, and they do not appear to produce pseudo functions without considerable manual modifications.

Here we approach the problem of generating the PRP curves as a nonlinear estimation problem. We refer to one of the papers by $\mathrm{T}$. Watson et al. ${ }^{6}$ where similar ideas are applied to estimate relative permeability and capillary pressure functions from laboratory displacement experiments. They used cubic splines as a representation of relative permeability functions and a regularization operator to cure any ill-posed nature of the estimation problem.

The use of a multi-resolution method based on an application of the Haar basis (multi-scale basis) was described by $\mathrm{J}$. Liu? ${ }^{7}$. It was shown that the method gives a natural regularization approach which is complementary to Tikhonov's regularization.

\section{PROBLEM STATEMENT}

Pseudo relative permeability (PRP) functions are generated to reproduce to a certain extent the results from a fine scale simulation on a coarse scale grid. In this sense they are in fact the scaled up original (rock) relative permeability curves.

The generation of PRP functions can be formulated as solving a nonlinear estimation problem. If we assume that a fine grid model with rock relative permeability curves gives us a correct answer (which we hereafter call 'data'), and with a specific parameterization procedure chosen for the PRP curves, then the PRP relationships should ensure the smallest obtainable deviation between data and coarse grid simulation results. Since we want the PRP to reproduce the results of the fine grid simulation, a variance error, which in our case is a numerical error of the fine grid results, can be neglected compared to a bias error (essentially the incapability of the coarse grid model to represent the true data).

As a measure of the deviation we use the least squares objective function

$$
J(u)=\frac{1}{2}(x(u)-y){ }^{T} C_{\mathrm{u}}^{-1}(x(u)-y),
$$

where vector $y=\left(y_{1}, \ldots, y_{\mathrm{m}}\right)$ is the data (the results of the fine grid model), $x(u)$ is the vector to be matched (the results of the coarse grid model, also with $m$ components), $u=\left(u_{1}, \ldots, u_{4}\right)$ is the vector of $q$ parameters to be estimated (representing the PRP curves), and $C_{u}$ is a weighting matrix.

Inequality constraints, requiring the PRP estimates to be monotone and stay in the range between 0 and 1 , can be put in the form

$$
g(u) \geq 0 \text {. }
$$

Equality constraints stating that $x(u)$ is a solution of the simulation problem is

$$
F(x(u), u)=0,
$$

where $F$ is the vector of simulator equations.

The goal is to find an estimate of the vector $u$ satisfying (2) and (3), while delivering a minimum to the objective function (1):

$$
u^{*}=\min _{u \in(2),(3)}(J(u))
$$

\section{MINIMISATION PROCEDURE}

The search has to be iterative because the objective function $J$ depends on the estimated PRP parameters in a strongly nonlinear manner. The expansion of the objective function up to the second order of accuracy around a given estimate $u$ is

$$
J(u+\Delta u)=J(u)+J_{\mathrm{u}}^{T} \Delta u+\frac{1}{2} \Delta u^{T} H \Delta u+o\left(\Delta u^{T} \Delta u\right),
$$

where $\Delta u$ is an increment of the vector $u, J_{u}^{T}$ is a gradient of the objective function, $J_{u}^{T}=\left(\frac{\partial J}{\partial u_{1}}, \ldots, \frac{\partial J}{\partial u_{4}}\right)$, and $H$ is its Hessian matrix, with elements $H_{\mathrm{ps}}=\frac{\partial^{2} J}{\partial u_{\mathrm{p}} \partial u_{\mathrm{s}}}$. Constraints (2) and (3) can be put as

$$
\begin{aligned}
& g(u+\Delta u) \geq 0, \\
& F(x(u+\Delta u), u+\Delta u)=0 .
\end{aligned}
$$

The equality constraint (7) provides an efficient way of calculating $J_{\mathrm{u}}^{T}$ and a sufficiently accurate estimate $H$. We shall return to this point later. The inequality constraint (6) can be linearised if necessary:

$$
g(u)+\frac{\partial g}{\partial u} \cdot \Delta u \geq 0
$$

Thus the original minimisation problem (1) - (4) has been reduced to an iterative sequence of simpler problems.

On each iteration the quadratic function (5) subject to the linear inequality constraint (8) is to be minimised. 
The succession of $\Delta u_{i}$ having been obtained on each iteration provides then an optimal trajectory from an original estimate of the vector $u$ towards its best estimate.

The solution for an unconstrained minimum of the function (5) is

$$
\Delta u=-H^{-1} J_{\mathrm{u}}^{T} \text {. }
$$

Then, the next estimate of $u$ can be found as

$$
u_{i+1}=u_{i}+\Delta u \text {. }
$$

With the surface of function (5) flattening out along certain directions it will reflect any ill-posed nature of the nonlinear minimisation problem (1) - (4). This kind of peculiarity often leads to the effect of $\Delta u$ and $u_{\mathrm{i}+1}$ violating the constraints, usually along the least sensitive directions. The simple remedy using 'cut off ' values deflects the search away from the optimal trajectory, thus slowing down the convergence rate dramatically and often destroying convergence completely.

Two different techniques have been used to solve the local minimisation problem (5), (8).

\section{Discrimination}

The idea is to determine the directions along which the variation of the function (5) is relatively large and to use them for a basis of a new subspace within which the search for minimum will be conducted ${ }^{8,9}$. Let $V$ be the matrix with columns being the normalised eigenvectors of $H$, and let the diagonal matrix $\Lambda$ contain the eigenvalues $\left(\lambda_{\mathrm{i}}\right)$. We shall set up a new matrix $V_{\mathrm{r}}$ containing a reduced set of the eigenvectors corresponding to the largest eigenvalues $\left(\lambda_{\mathrm{r}}\right)$. Then the reduced vector $\Delta \tilde{u}=\left(\Delta \tilde{u}_{1}, \ldots, \Delta \tilde{u}_{\mathrm{r}}\right), r<q$, is defined by

$$
\Delta u=V_{\mathrm{r}} \Delta \tilde{u}
$$

The solution of the problem (5), (8), in the transformed coordinates, can be obtained by substituting (11) into (5):

$$
\Delta \tilde{u}=-\left(\Lambda_{\mathrm{r}}^{2}\right)^{-1} V_{\mathrm{r}}^{T} J_{\mathrm{u}}
$$

Since the search is carried out along the sensitive directions only, unstable components are removed from the solution. A minor adjustment of the back transformation is usually sufficient to meet the constraint (8):

$$
\Delta u=\psi V_{\mathrm{r}} \Delta \tilde{u},
$$

where $\psi \leq 1$ is a tuning factor.

\section{Constrained Minimization}

We modify the function (5) by adding a penalty term accounting for the constraint (8). The modified objective function is

$$
\tilde{J}(u+\Delta u)=J(u+\Delta u)+G^{T}(u, \Delta u) B G(u, \Delta u),
$$

where $B$ is the diagonal matrix of the penalty weights and $G(u, \Delta u) \equiv g(u)+\frac{\partial g}{\partial u} \cdot \Delta u$.

follows:

The elements $b_{\mathrm{ii}}$ of the matrix $B$ are defined as

$b_{\mathrm{ii}}=\left\{\begin{array}{l}0, \text { if } \mathrm{i} \text {-th constraint is true, } \\ \text { (a large value), }\end{array}\right.$
The conjugate gradient technique is then applied to minimise the modified function (14). The use of sufficiently large values of the penalty weights ensures that the solution $\Delta u$ does not violate the constraint (8).

\section{Calculation of the Gradient and the Hessian Matrix}

The expansion of the objective function (1) up to the second order of accuracy yields the following expression:

$$
\begin{aligned}
J(u, \Delta u)= & J(u)+\left(e^{T} C_{\mathrm{u}}^{-1} \frac{\partial x}{\partial u}\right) \Delta u+\frac{1}{2} \Delta u^{T}\left(\frac{\partial x}{\partial u}\right)^{T} C_{\mathrm{u}}^{-1} \frac{\partial x}{\partial u} \Delta u \\
& +\frac{1}{2} e^{T} C_{\mathrm{u}}^{-1} \Delta u^{T} \frac{\partial^{2} x}{\partial u^{2}} \Delta u+o\left(\Delta u^{T} \Delta u\right)
\end{aligned}
$$

where $e=x(u)-y$ is a residual vector.

The fourth term in the expansion involves calculating the second derivatives of the vector $x$, which is prohibitively expensive. General experience, though, has shown that in most cases the term is relatively small and can be neglected. In that case, the only quantity that needs to be determined is $\partial x / \partial u$, which is usually called sensitivity matrix.

$$
\begin{aligned}
& \text { Linearization of Eq. (7) yields } \\
& \qquad \frac{\partial F}{\partial x}\left(\frac{\partial x}{\partial u}\right)=-\frac{\partial F}{\partial u},
\end{aligned}
$$

where $\partial F / \partial x$ is the Jacobi matrix of the simulator equations. Thus, to obtain the sensitivity matrix $\partial x / \partial u$ we have to solve $q$ sets of linear equations for each of the vectors $\partial x / \partial u_{1}, \ldots, \partial x / \partial u_{q}$. The overall CPU time spent on obtaining an estimate of the vector $u$, depends strongly on efficiency of the linear solver used. In fact, the Eq. (16) puts the most critical restriction on the number of estimated parameters with respect to a CPU capacity available. The restriction becomes much weaker if the simulator problem has a moderate size and direct methods can be used to solve the Eq. (16).

For more comprehensive discussions consult References 8 to 11 .

\section{Parameterization of the PRP Functions}

The parameterization of the PRP curves has been done to convert a function estimation problem to a parameter estimation problem. The guidelines for selecting a suitable representation were: a) simplicity; b) direct interpretation of results; c) robustness; d) direct communication with the commercial simulator used (Eclipse). Then, the optimal choice is to define adjustable parameters to be relative permeability values at certain specified saturation knots, while PRP relationships are being determined as a linear interpolation between corresponding knots. The inequality constraint, which is linear in the parameters, can easily be derived.

\section{CROSS-SECTIONAL MODELS}

For the Fangst (F), Tilje (T), and Aare (Aa) formations of the Heidrun field, all from the Jura period, PRP functions have been constructed, by using 2D crosssectional numerical models that are assumed to represent the entire horizontal extension of the layers. 
Due to some distinct barriers to vertical flow the reservoir was divided into 5 sub-reservoirs corresponding to formation groups defined for the Heidrun: F5, F1-F3, T3-T4, Aa2\&T1, and Aa1B. The group F1-F3 included the 5 layers F1B, F2, F3A, F3B, and F3C. The formation T3 embodied 3 layers T3A, T3B, T3C, and Aa2 was constituted of $\mathrm{Aa} 2 \mathrm{~A}$ and $\mathrm{Aa} 2 \mathrm{~B}$. For each of the 5 subreservoirs pseudo function sets were generated independently. In total 14 of the original model layers were studied.

In all simulations a water injection well was located near the down dip end of the cross-section and an oil producing well was placed close to the up dip end. The wells were assumed to be vertical and to be perforated and connected to all layers.

The models have been modified into a fine scale version by subdividing blocks, and thus making the horizontal block length approximately 10 to $14 \mathrm{~m}$, and by introducing a refined layering based on contrasts in the horizontally directed absolute permeability $\left(K_{x y}\right)$ at representative wells. The principal assumption behind this procedure is that horizontal permeability variations on the fine scale level will allow flooding behaviour to depart in a decisive way from what would be calculated with the original coarse grid model. For the reference case the rock curves were used.

Consulting well logs the boundaries between the sublayers were placed at positions along the well trajectory where $K_{\mathrm{xy}}$ changed rather abruptly. Within each of these sublayers also other parameters retrieved, ie the vertical absolute permeability $\left(K_{\mathrm{z}}\right)$, net-to-gross ratio, porosity, and initial water saturation are fairly uniform. For every sublayer an arithmetic average of the parameter is estimated from log data, except for the $K_{7}$, which is taken as a geometric average. The coarse scale layers were divided vertically along the entire cross-section length into sublayers.

The parameters of the coarse grid models are determined by recombining the sublayers. For the recombined layers the average vertical permeability is calculated as the harmonic mean, while the other parameters taken to be arithmetic mean values. The grid data for all simulation runs, both fine (F) and coarse (C) cases, are summarised in Tab. 1 in the Appendix.

From a comprehensive investigation of the relative permeabilities for the Heidrun field, certain relationships for these parameters as functions of normalised water saturation $S_{\mathrm{wn}}$, using Corey exponents $\alpha$ and $\beta$, are recommended:

with

$$
\begin{aligned}
& K_{\mathrm{rw}}=K_{\mathrm{rw}}\left(S_{\mathrm{orw}}\right) \times S_{\mathrm{wn}}^{\alpha}, \\
& K_{\mathrm{ro}}=\left(1-S_{\mathrm{wn}}\right)^{\beta}, \\
& S_{\mathrm{wn}}=\left(S_{\mathrm{w}}-S_{\mathrm{wi}}\right) /\left(1-S_{\mathrm{wi}}-S_{\mathrm{orw}}\right) .
\end{aligned}
$$

where $S_{w i}$ is the initial water saturation and $S_{o r w}$ is residual oil saturation anticipated after water flooding. These are the rock curves, and they have been determined by analysing the results from laboratory experiments where water was forced to displace oil in selected plugs drilled out of core samples taken from the field. The parameters
$S_{\text {orw }}, K_{\mathrm{rw}}\left(S_{\text {orw }}\right), \alpha$, and $\beta$ are specified in Tab. 2 in the Appendix.

The water injection rate was adjusted to allow production with an acceptable water cut for 6 to 15 years and a plateau lasting around 2 to 4 years.

\section{RESULTS AND DISCUSSION}

Two production parameters, water cut (WWCT) and bottomhole pressure (WBHP) in the production well, were used to estimate the PRP curves. Since WBHP in the injection well has been kept constant, the overall pressure drop between the producer and the injector has been matched as well. The minimisation procedure based on the discrimination technique has been used for all layers, and for Aa1B also the constrained minimisation has been applied.

The derived PRP curves allowed for the simulation of a more gradual displacement which on average was much closer to the corresponding fine grid case than the more piston-like front movement described by coarse grid simulations with the rock curves. By not introducing PRP functions for the coarse grid the water front has been predicted substantially too early for F5 and too late for F1-F3, Aa2\&T1 and Aa1B.

The parameters for 9 layers, with relative permeability functions defined in terms of Corey exponents, are presented in Tab. 3. For the remaining 5 layers (T3B, T1, Aa2B, Aa2A, Aa1B) the pseudo relative permeability functions were obtained pointwise, and these functions are depicted in Figs. 1-4 and 13 in the Appendix.

The most interesting case of all having been considered is probably Aa1B. The cross-sectional fine grid model of the layer is shown in Fig. 5. The layer is very heterogeneous with a permeability contrast around 100 . There are no barriers within Aa1B to prevent vertical flow to occur even though the vertical permeability for some sublayers is fairly low. The coarsening of the Aa1B fine grid model has been the most drastic of all the cases. The fine grid model has been scaled up to the coarse grid consisting of only one layer (Fig. 6).

The coarse grid simulation using the rock relative permeability, curves resulted in significantly later water breakthrough time (Fig. 8 ) and $14 \%$ higher cumulative oil production, compared to the fine grid simulation (Fig. 7).

Several sets of PRP functions have been generated for the coarse grid model using both the discrimination and the constrained minimisation techniques. About 10 knots in all have been used to represent the PRP curves except for one case where the number of the knots has been halved. The locations of the knots have been determined by examining the eigenvalue decomposition of the Hessian matrix in Eq. (9), or rather its estimate in Eq. (15). The knots have been rearranged along the saturation axis to cover the segments where the objective function is most sensitive.

The derived PRP curves have delivered almost perfect fit of the cumulative oil production by both minimisation techniques $(0.02 \%$ and $0.3 \%$ for the constrained 
minimisation and the discrimination, respectively). The corresponding profiles can be seen in Fig. 7.

The WWCT plots are shown in Fig. 8. The WWCT profile from the coarse grid model with PRP functions obtained by the discrimination procedure, reproduces the fine grid results nicely, although it is incapable of capturing its peculiar shape in detail. The use of the PRP curves generated by the constrained minimisation procedure results in an almost perfect match. In this case the WWCT development shows two distinct branches corresponding to the water arriving consecutively from the two most permeable layers (see Fig. 5).

The plots illustrating the final fit of the oil production rate and WBHP in the producing well are presented in Figs. 9 and 10, respectively.

The most attractive and promising feature of the constructed PRP functions is that they not only allow for reproducing local quantities like WWCT, WBHP, etc at certain points, but also give a general picture of the flow and distributed properties, for instance water saturation. Figs. 11 and 12 show the comparison between vertically averaged saturation profiles from the fine grid model and corresponding profiles from the coarse grid model. One of the profiles was obtained by using the rock curves also for coarse grid, and the other with PRP functions generated by constrained minimisation procedure. It is clearly seen from Fig. 11 that the use of PRP curves results in a very good match.

Fig. 13 shows the rock relative permeability curves and two sets of PRP relationships that have been generated by using the two minimisation techniques. As it could be expected, the PRP curves for the water are convex upward, reflecting the very disperse average water saturation profiles from the fine grid model. The progress of the constrained minimisation procedure, illustrating its convergence behaviour, is presented in Fig. 14.

Robustness of the generated sets of the PRP curves has been tested by comparing the fine and coarse grid simulations that have been run with the oil production rate equal to 0.5 and 1.5 of originally used. In both cases the PRP functions have delivered a very good fit, which is considerably better than one obtained by using the rock curves.

\section{CONCLUSIONS}

The nonlinear regression based technique has been developed and successfully applied to generate pseudo relative permeability curves for several heterogeneous layers of the Heidrun field.

Two different minimisation techniques have been used. Both proved to be robust and reliable for practical applications, with the constrained minimisation giving a better fit than the discrimination technique.

The simple piecewise linear representation of the relative permeability curves with a rather moderate number of knots allows a very accurate reproduction of the fine grid simulation results, even in the case of a strongly heterogeneous reservoir.
Derived pseudo relative permeability functions proved to be valid within a wide range of production rates and therefore provide reliable basis for a full field simulation.

The developed procedure is anticipated to be a good tool for scaling up multiphase flow models.

\section{NOMENCLATURE}

PRP - pseudo relative permeability

$J$ - objective function

$x$ - result of the coarse grid model (vector)

$y$ - result of the fine grid model (vector)

$u$ - vector of parameters to be estimated

$C_{\mathrm{u}}$ - weighting matrix

$g$ - vector of inequality constraints

$F$ - vector of simulator equations

$u^{*}$ - best estimate of vector $u$

$J_{u}$ - gradient of the objective function

$H$ - Hessian matrix of the objective function

$\Delta u$ - increment of the current estimate $u$ (vector)

$\partial g / \partial u$ - Jacobi matrix of $g$ with respect to $u$

$V$ - matrix containing the normalised eigenvectors of $H$ in its columns

$\Lambda$ - diagonal matrix with the eigenvalues of $H$

$V_{\mathrm{r}}$ - reduced matrix $V$

$\Lambda_{r}$ - reduced matrix $\Lambda$ containing largest eigenvalues

$\Delta \tilde{u}$ - transformed vector increment

$\psi$ - tuning parameter

$G$ - penalty matrix

$B$ - matrix of penalty weights

$o(.$.$) - order of approximation$

$e$ - residual vector

$\partial F / \partial x$ - Jacobi matrix of simulator equations

$\partial x / \partial u$ - sensitivity matrix

$K_{x y}$ - horizontally directed absolute permeability

$K_{\mathrm{z}}$ - vertically directed absolute permeability

$K_{r w}$ - relative permeability for water

$K_{\mathrm{ro}}$ - relative permeability for oil

$S_{w}$ - water saturation

$S_{\text {wi }}$ - initial water saturation

$S_{\mathrm{wn}}$ - normalised water saturation

$S_{0}$ - oil saturation

$S_{\text {orw }}$ - residual oil saturation

$\alpha, \beta$ - Corey exponents

Upper index $T$ for the transpose $A^{T}$ of matrix $A$

\section{ACKNOWLEDGEMENTS}

The authors very much express their appreciation with thanks to Conoco Norway Inc., Neste Petroleum A/S, and Den norske stats oljeselskap a.s (Statoil) for the kind permission to publish this paper.

\section{REFERENCES}

(1) Stiles, W. E. (1949): Use of Permeability Distribution in Water Flood Calculations. Trans. AIME, 186, 9-13.

(2) Hearn, C. L. (1971): Simulation of Stratified Waterflooding by Pseudo Relative Permeability Curves. J. Pet. Tech., July, 805-813. 
(3) Thomas, G. W. (1983): An Extension of Pseudofunction Concepts. SPE 12274, 415-423 ( + additional pages with figures 2-9).

(4) Dake, L. P. (1978): Fundamentals of Reservoir Engineering. Elsevier, $443 \mathrm{pp}$.

(5) Mattax, C. C. and R. L. Dalton (1990): Reservoir Simulation. SPE Monograph, 13, 173 pp.

(6) Watson, A. T., P. C. Richmond, P. D. Kerig, and T. M. Tao (1988): A Regression - Based Method for Estimating Relative Permeabilities from Displacement Experiments. SPE Reservoir Engineering, Aug., 953-958.

(7) Liu, J. (1993): A Multiresolution Method for Distributed Parameter Estimation. SIAM J. Sci. Comput., Vol. 14, No. 2, 389-405.

(8) Shah, P. C., G. R. Gavalas, and J. H. Seinfeld (1978): Error Analysis in History Matching: The Optimum level of Parametrization. SPEJ, June, 219-228.

(9) Duijndam, A. J. W. (1988): Bayesian Estimation in Seismic Inversion. Part I: Principles. Geophysical Prospecting, 36, 878-898. (Part II: Uncertainty analysis, pp. 899-918.)

(10) Gavalas, G. R., P. C. Shah, and J. H. Seinfeld (1976): Reservoir History Matching by Bayesian Estimation. SPEJ, Dec., 337-350.

(11) Anterion, F., R. Eymard, and B. Karcher (1989): Use of Parameter Gradients for Reservoir History Matching. SPE 18433, SPE Symp. on Res. Sim., Houston, Feb. 6-8, 339-354.

\section{APPENDIX: TABLES AND FIGURES}

Table 1: Grid data for all simulation runs, fine $(F)$ and coarse (C) cases, with the number of blocks between the injector and the producer, $N_{\mathrm{I}-\mathrm{P}}$, and the average block length $\Delta Y_{\mathrm{I}-\mathrm{P}}$.

\begin{tabular}{|c|ccrrc|}
\hline Task & F/C & $\begin{array}{c}\text { Grid dimensions } \\
\left(N_{\mathrm{x}} \times N_{\mathrm{y}} \times N_{\mathrm{z}}\right)\end{array}$ & $\begin{array}{r}\text { No. of } \\
\text { blocks }\end{array}$ & $N_{\text {I.P }}$ & $\begin{array}{c}\Delta Y_{\text {I-P }} \\
(\mathrm{m})\end{array}$ \\
\hline F5 & $\mathrm{F}$ & $1 \times 309 \times 34$ & 10506 & 240 & 10 \\
& $\mathrm{C}$ & $1 \times 38 \times 1$ & 38 & 28 & 86 \\
\hline F1 - F3 & $\mathrm{F}$ & $1 \times 300 \times 35$ & 10500 & 240 & 10 \\
& $\mathrm{C}$ & $1 \times 36 \times 5$ & 180 & 28 & 87 \\
\hline T3 - T4 & $\mathrm{F}$ & $1 \times 255 \times 27$ & 6885 & 195 & 12 \\
& $\mathrm{C}$ & $1 \times 36 \times 4$ & 144 & 27 & 90 \\
\hline Aa2\&T1 & $\mathrm{F}$ & $1 \times 245 \times 23$ & 5635 & 182 & 13.6 \\
& $\mathrm{C}$ & $1 \times 35 \times 3$ & 105 & 27 & 95 \\
\hline Aa1B & $\mathrm{F}$ & $1 \times 315 \times 13$ & 4095 & 245 & 13.6 \\
& $\mathrm{C}$ & $1 \times 45 \times 1$ & 45 & 35 & 95 \\
\hline
\end{tabular}

Table 2: Parameters for the rock curves.

\begin{tabular}{|c|cccc|}
\hline Formation or zone & $S_{\text {orw }}$ & $K_{\mathrm{rw}}\left(S_{\mathrm{orw}}\right)$ & $\alpha$ & $\beta$ \\
\hline F5 & 0.3 & 0.4 & 4 & 2.0 \\
F1 - F3 & 0.3 & $\left(^{*}\right)$ & 2 & 2.2 \\
Tilje & 0.2 & $\left(^{*}\right)$ & 2 & 2.5 \\
Aare & 0.25 & 0.45 & 2 & 2.5 \\
\hline
\end{tabular}

$\left.{ }^{*}\right)$ Stepwise functions $K_{\text {rw }}\left(S_{\text {orv }}\right)$ versus $K$ for F1-F3 and Tilje:

\begin{tabular}{|c|c|c|c|}
\hline \multicolumn{2}{|c|}{$F 1-F 3$} & \multicolumn{2}{|c|}{ Tilje } \\
\hline$K$ - range $(\mathrm{mD})$ & $K_{r w}\left(S_{o r w}\right)$ & $K-$ range (mD) & $K_{r_{w}}\left(S_{\text {orw } w}\right)$ \\
\hline $0-200$ & 0.12 & $0-30$ & 0.20 \\
\hline $200-700$ & 0.20 & $30-150$ & 0.37 \\
\hline $700-2000$ & 0.27 & $150-400$ & 0.48 \\
\hline $2000-6000$ & 0.32 & $400-800$ & 0.55 \\
\hline $6000-20000$ & 0.38 & 800 & 0.60 \\
\hline $20000-$ & 0.46 & & \\
\hline
\end{tabular}

(The stepwise functions approximate linear relations between $K_{r w}\left(S_{\text {or } w}\right)$ and $\log K$ that previously have been established.)

Table 3: Parameters of pseudo and rock curves recommended for the full-field model layers.

\begin{tabular}{|c|llll|}
\hline $\begin{array}{c}\text { Formation or } \\
\text { zone }\end{array}$ & Pseudo/Rock & $K_{\mathrm{rw}}\left(S_{\mathrm{urw}}\right)$ & $\alpha$ & $\beta$ \\
\hline F5 & Pseudo & 1.0 & 2.66 & 1.06 \\
F3C & Pseudo $(\$)$ & 0.301 & 1.79 & 1.904 \\
F3B & Pseudo $(\$)$ & 0.0274 & 2.0 & 1.9 \\
F3A & Rock (\$) & 0.12 & 2.0 & 2.2 \\
F2 & Rock & 0.12 & 2.0 & 2.2 \\
F1B & Pseudo & 0.336 & 2.0 & 2.06 \\
T4 & Rock & 0.2 & 2.0 & 2.5 \\
T3C & Rock & 0.4458 & 2.0 & 2.5 \\
T3A & Rock & 0.286 & 2.0 & 2.5 \\
\hline
\end{tabular}

(\$) For the task F1-F3 the final matching was done with an additional modification of $K_{\mathrm{z}}$ for the 3 layers F3C, F3B, and F3A, along with implementing the pseudo relative permeability curves for $F 3 C, F 3 B$, and F1B. While in the fine grid reference case the original relation was $K_{\mathrm{z}, 0 \mathrm{~g} g}=0.132 \times K_{\mathrm{xy}}$ for $\mathrm{F} 3$, the modified coarse grid simulation with pseudo curves also requires that we take $K_{\text {zmod }}=0.0224 \times K_{x y}$. Thus $K_{\mathrm{z}, \text { mod }}=0.17 \times K_{\text {zonig }}$ for $\mathrm{F} 3$. 


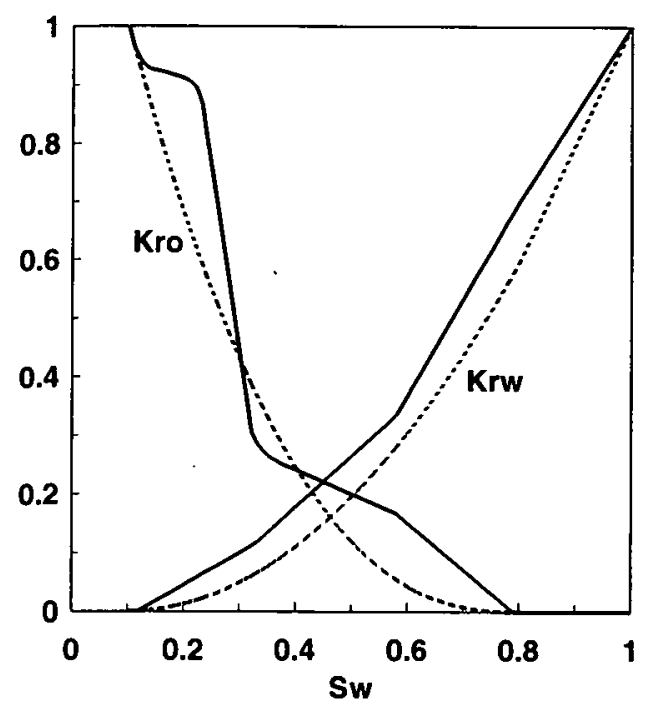

Figure 1: T3B rock and PRP functions.

Pseudo $\left(\_\right.$): Krw $=\mathrm{Krw}(\mathrm{Sw})$ and

$\mathrm{Kro}=\mathrm{Kro}(\mathrm{Sw})$, pointwise matched.

$\operatorname{Rock}(\ldots .):. \operatorname{Krw}(\mathrm{Swn})=0.5958 *$ Swn**2

$\mathrm{Kro}(\mathrm{Swn})=(1-\mathrm{Swn}) * * 2.5$, with

$S w n=(S w-0.1) /(0.8-0.1)$.

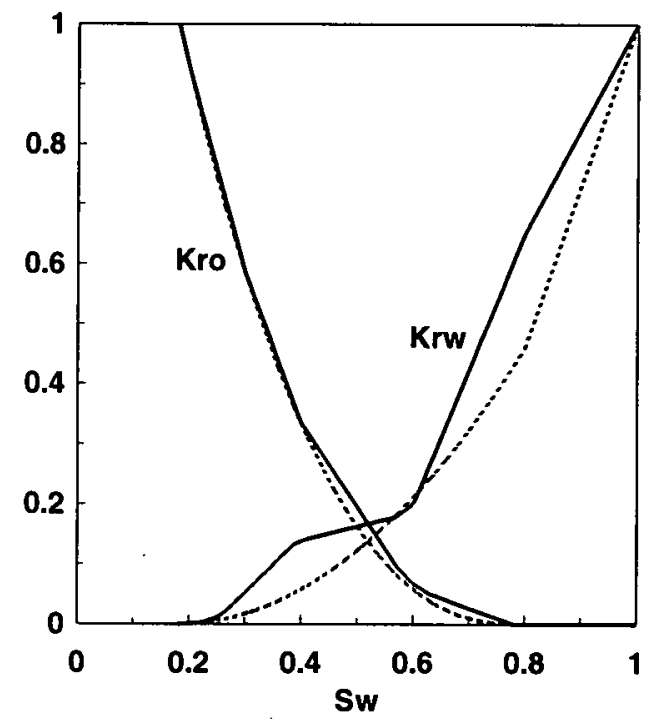

Figure 2: T1 rock and PRP functions.

Pseudo $\left(\_\right)$: Krw $=\mathrm{Krw}(\mathrm{Sw})$ and

$\mathrm{Kro}=\mathrm{Kro}(\mathrm{Sw})$, pointwise matched.

$\operatorname{Rock}(\ldots . .):. \operatorname{Krw}(\mathrm{Swn})=0.4594 * \mathrm{Swn}^{* * 2}$,

$\mathrm{Kro}(\mathrm{Swn})=(1-\mathrm{Swn})^{* *} 2.5$, with

$S w n=(S w-0.18) /(0.8-0.18)$.

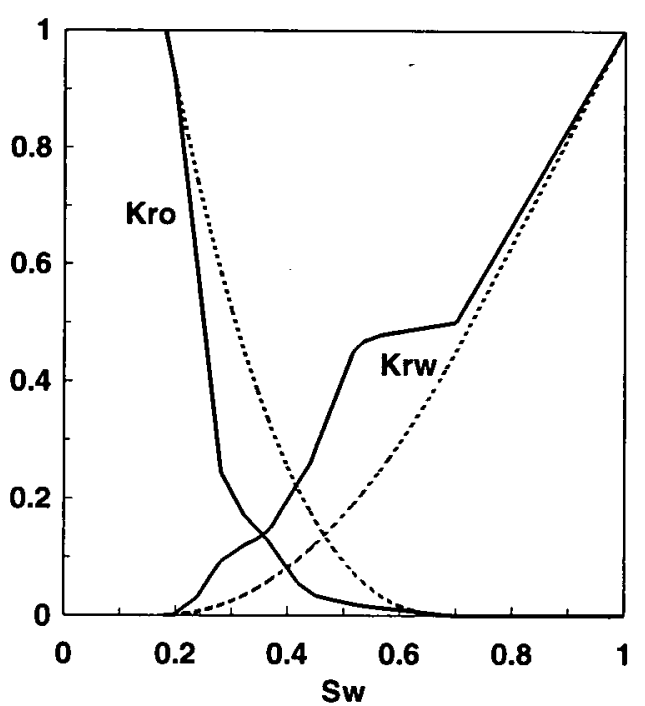

Figure 3: Aa2B rock and PRP functions.

Pseudo $(\ldots): \mathrm{Krw}=\mathrm{Krw}(\mathrm{Sw})$ and

$\mathrm{Kro}=\mathrm{Kro}(\mathrm{Sw})$, pointwise matched.

$\operatorname{Rock}(\ldots .):. \operatorname{Krw}(S w n)=0.45 * S w n * * 2$,

$\operatorname{Kro}(\mathrm{Swn})=(1-\mathrm{Swn}) * * 2.5$, with

$\mathrm{Swn}=(\mathrm{Sw}-0.18) /(0.7-0.18)$.

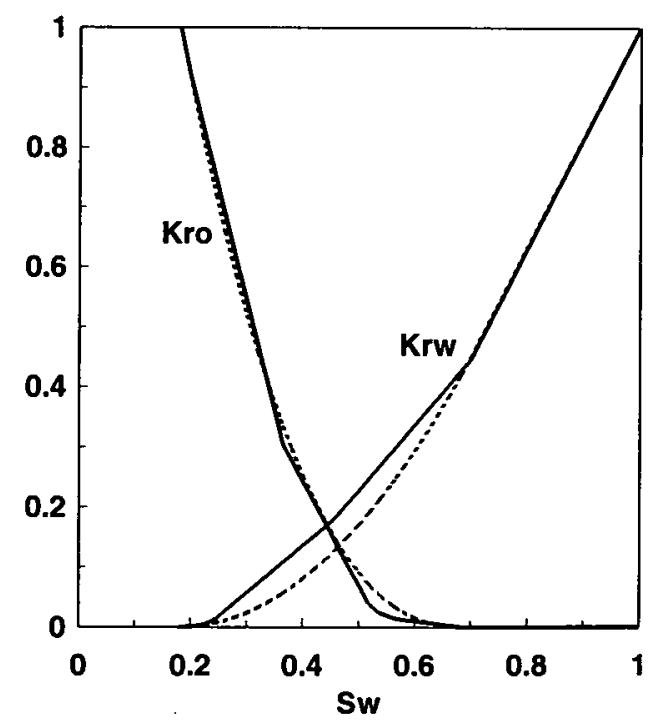

Figure 4: Aa2A rock and PRP functions.

Pseudo $(\ldots): \mathrm{Krw}=\mathrm{Krw}(\mathrm{Sw})$ and

$\mathrm{Kro}=\mathrm{Kro}(\mathrm{Sw})$, pointwise matched.

$\operatorname{Rock}(\ldots .):. \mathrm{Krw}(\mathrm{Swn})=0.45 * \mathrm{Swn}^{* * 2}$,

$\operatorname{Kro}(\mathrm{Swn})=(1-\mathrm{Swn})^{* *} 2.5$, with

$S w n=(S w-0.18) /(0.7-0.18)$. 


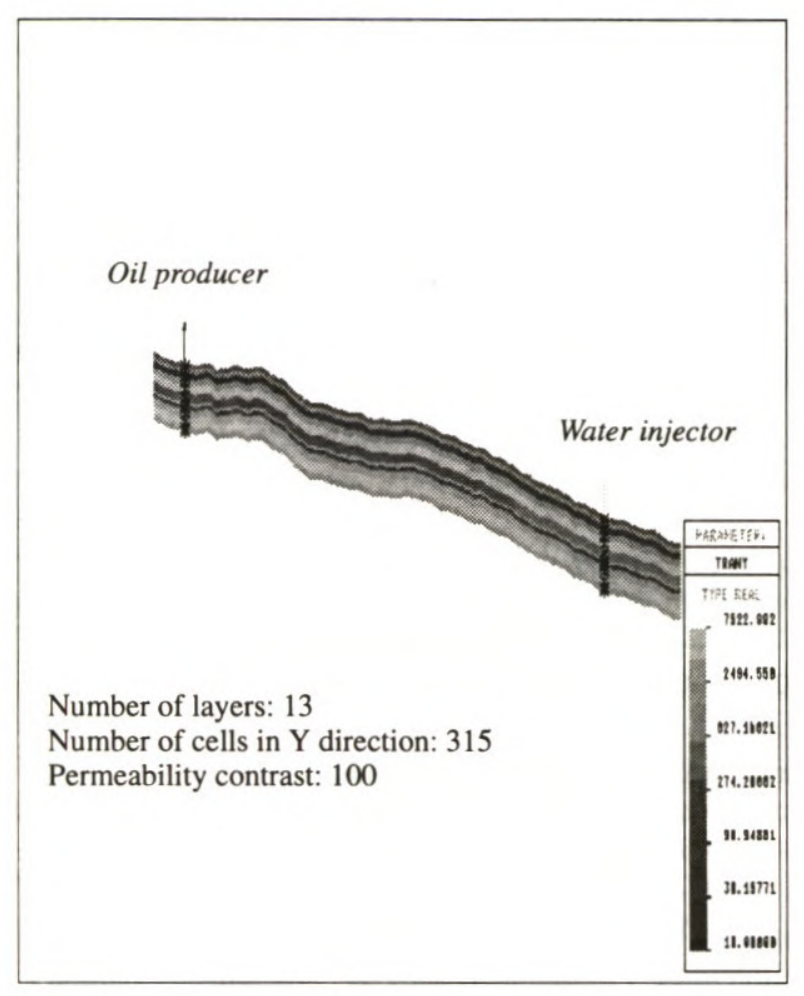

Figure 5: Fine scale model of the Aa1B layer.

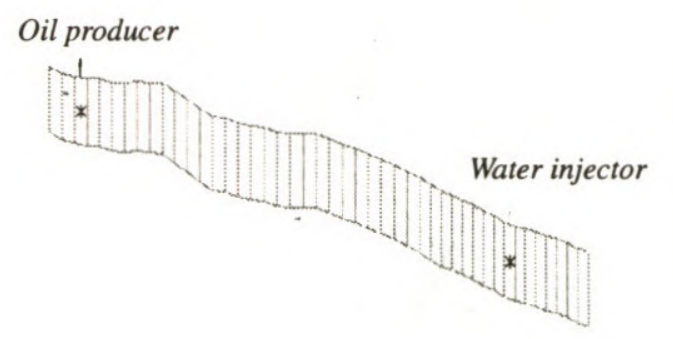

Number of layers: 1

Number of cells in Y direction: 35

Averaged permeability

Figure 6: Coarse scale model of the Aa1B layer.

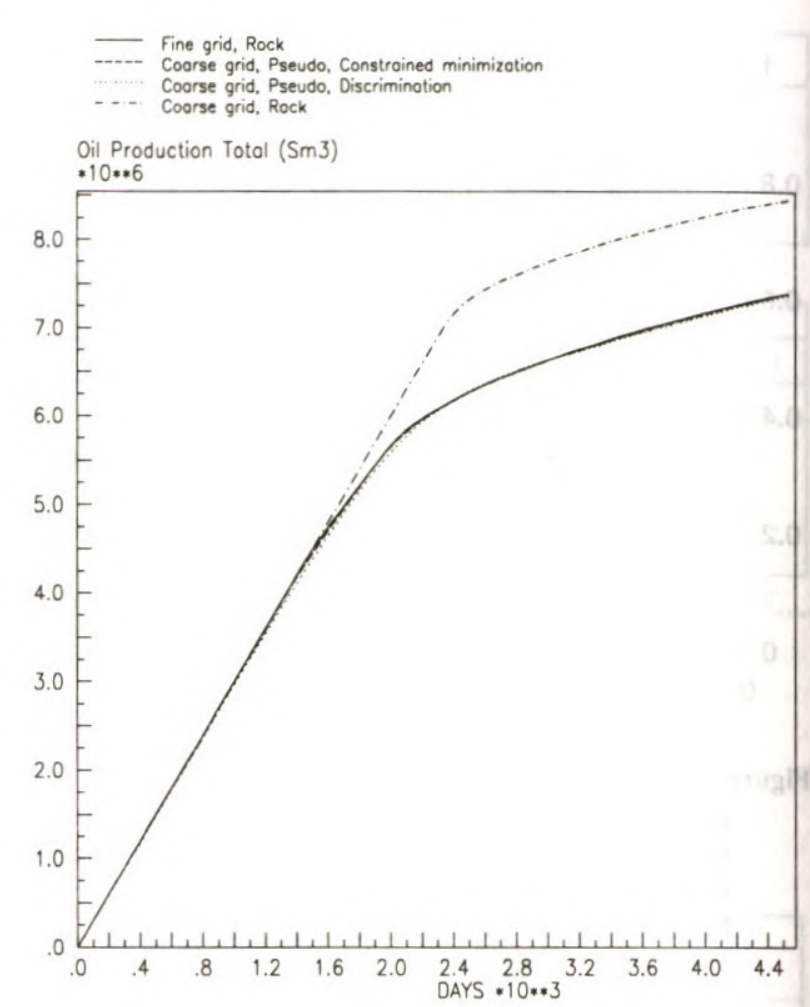

Figure 7: Cumulative oil production versus time.

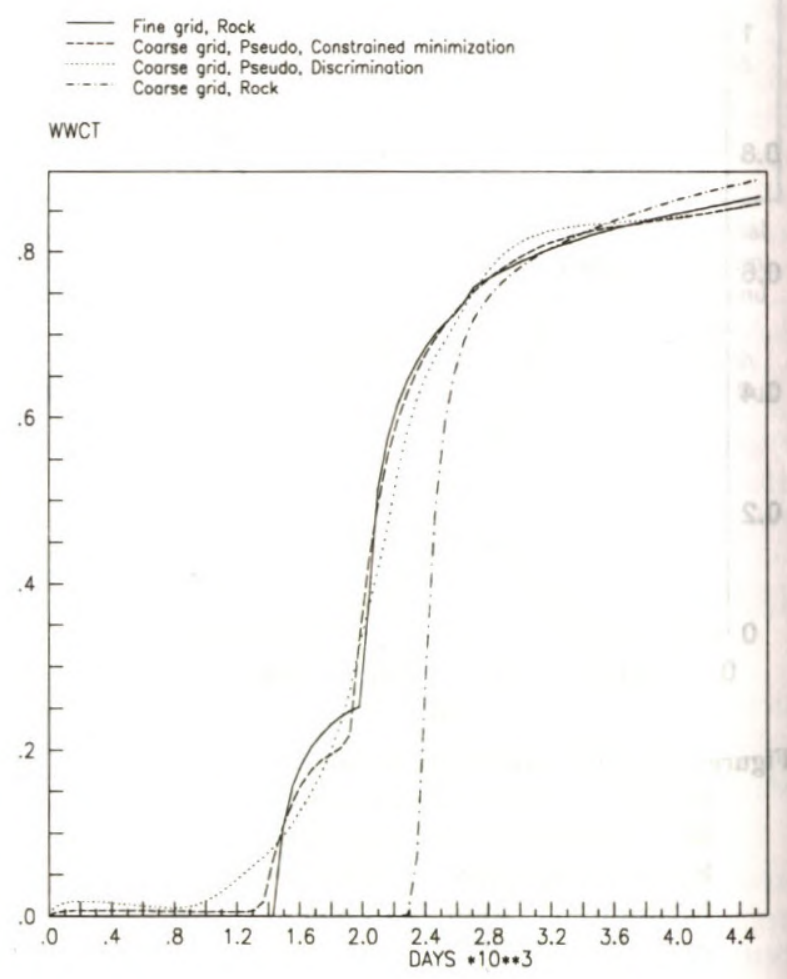

Figure 8: Watèr cut development versus time. 


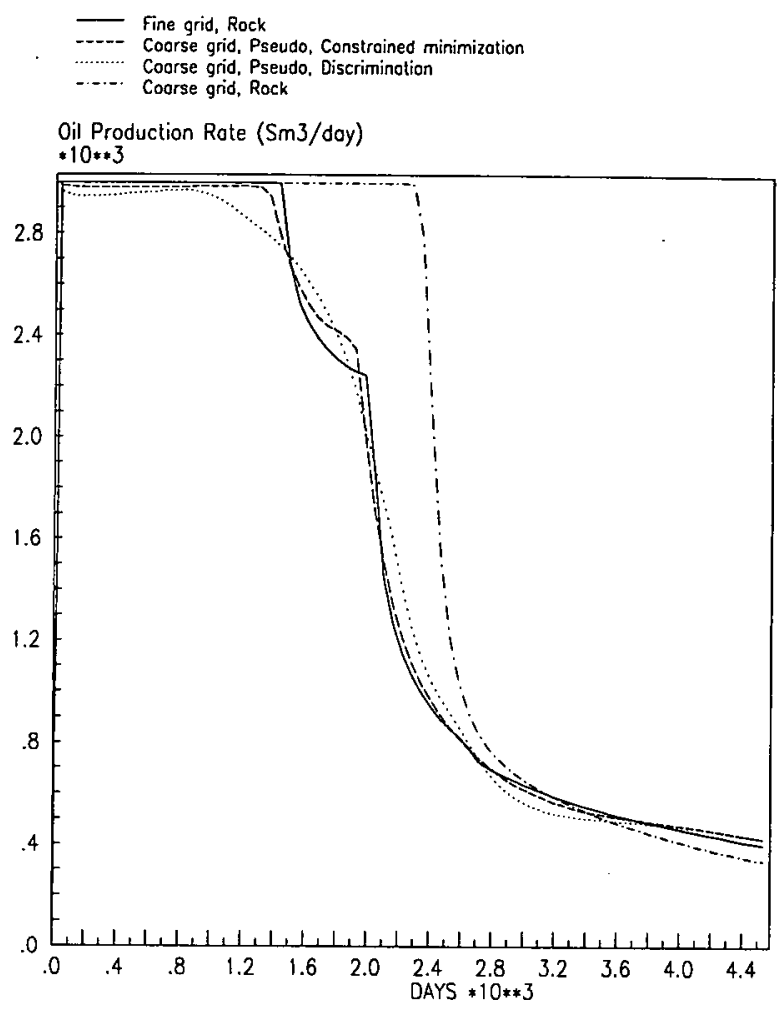

Figure 9: Oil rate versus time.

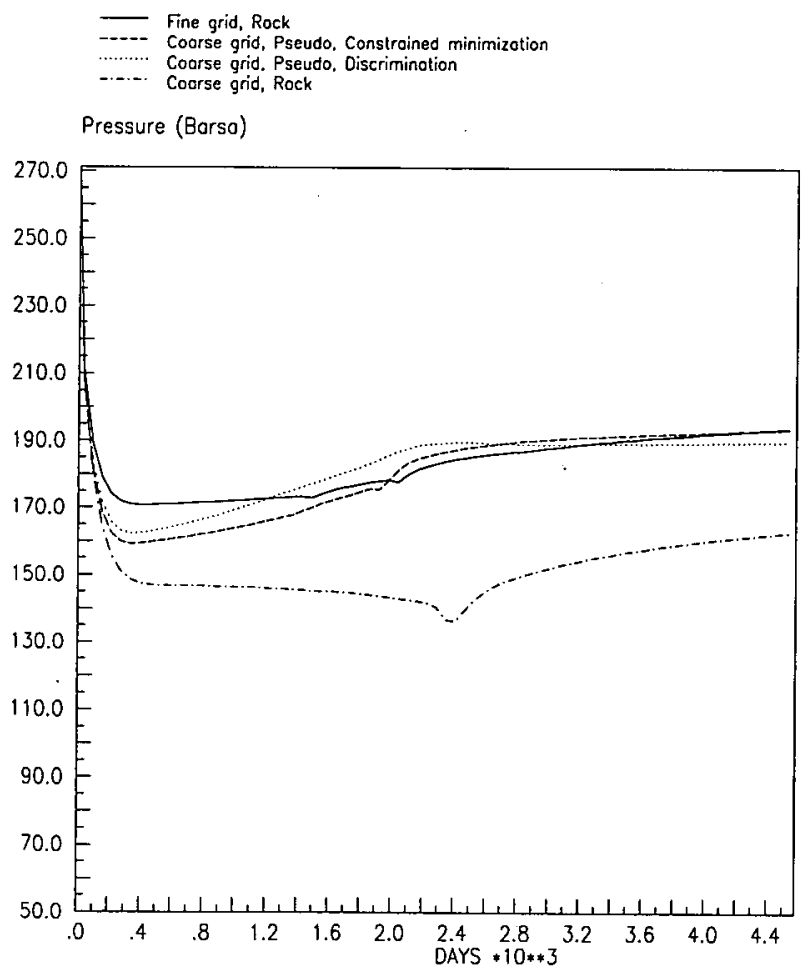

Figure 10: Bottomhole pressure in producing well.

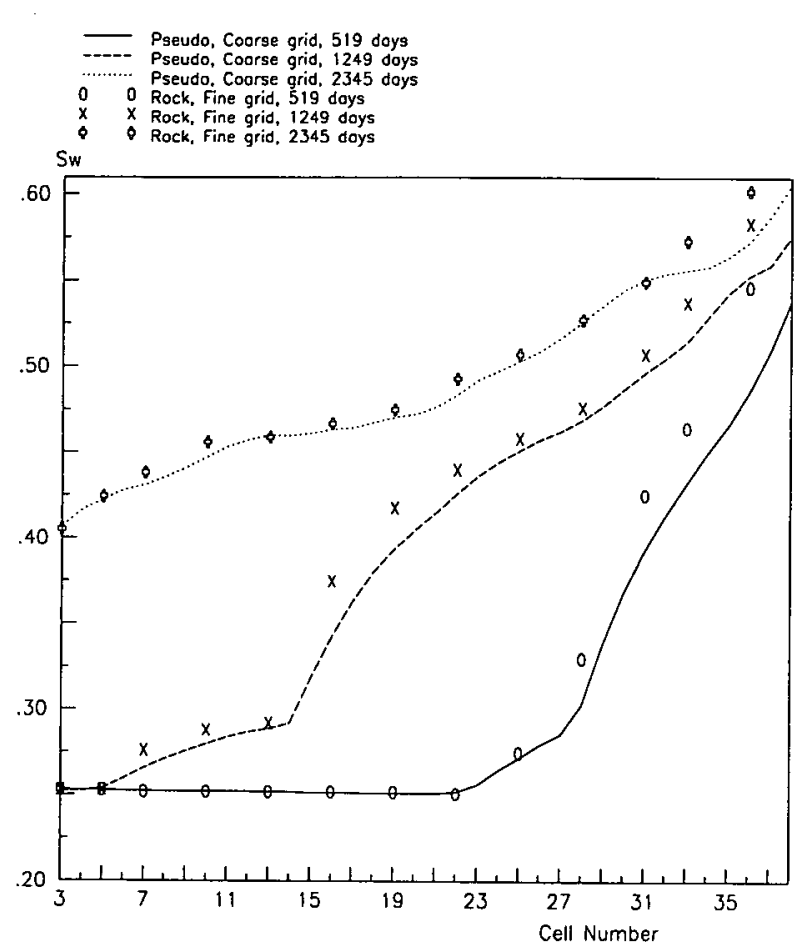

Figure 11: Water saturation distribution by cell number. PRP curves are used in coarse scale model.

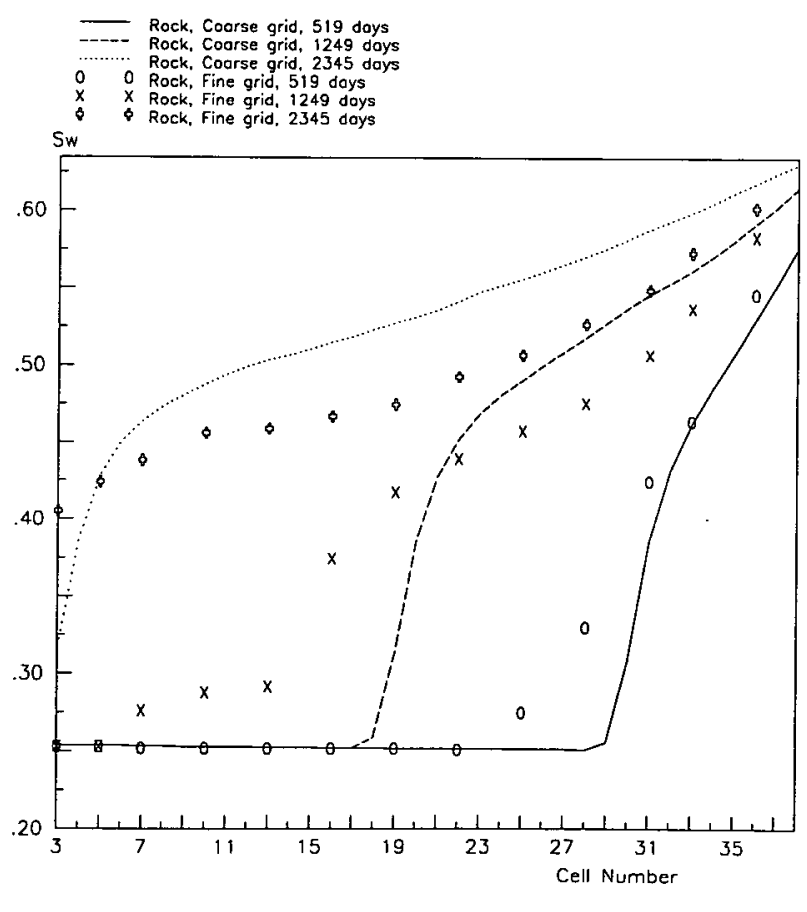

Figure 12: Water saturation distribution by cell number. Rock relative permeability curves are used in coarse scale model. 


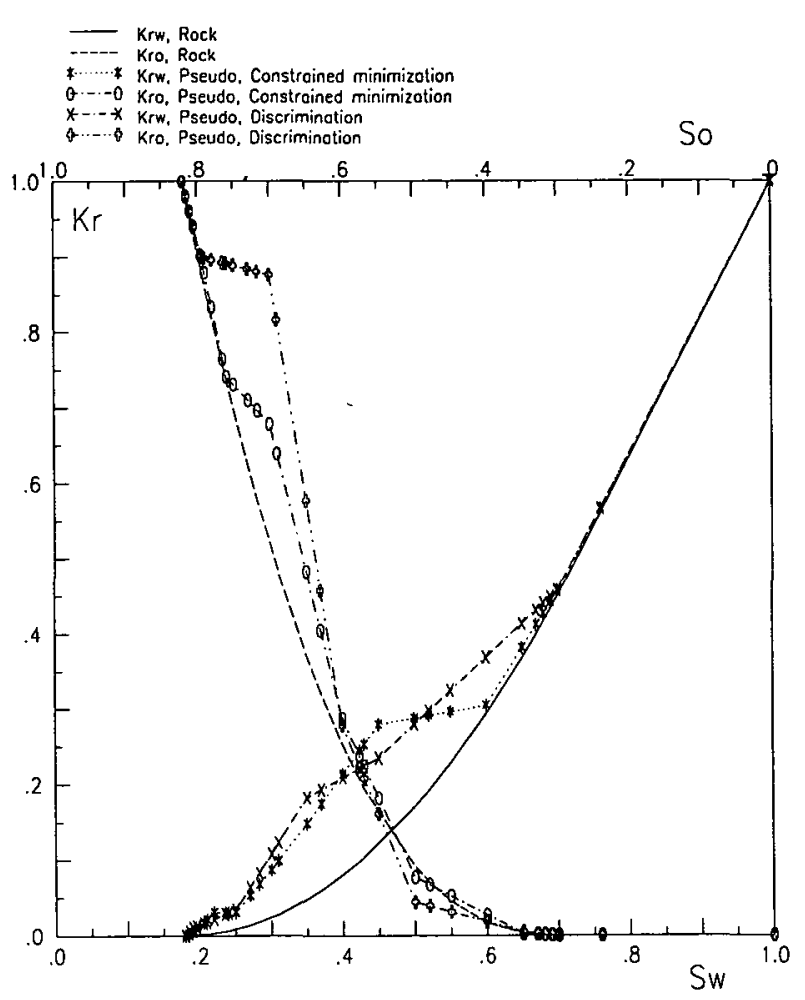

Figure 13: Rock and PRP functions for the Aa1B layer.

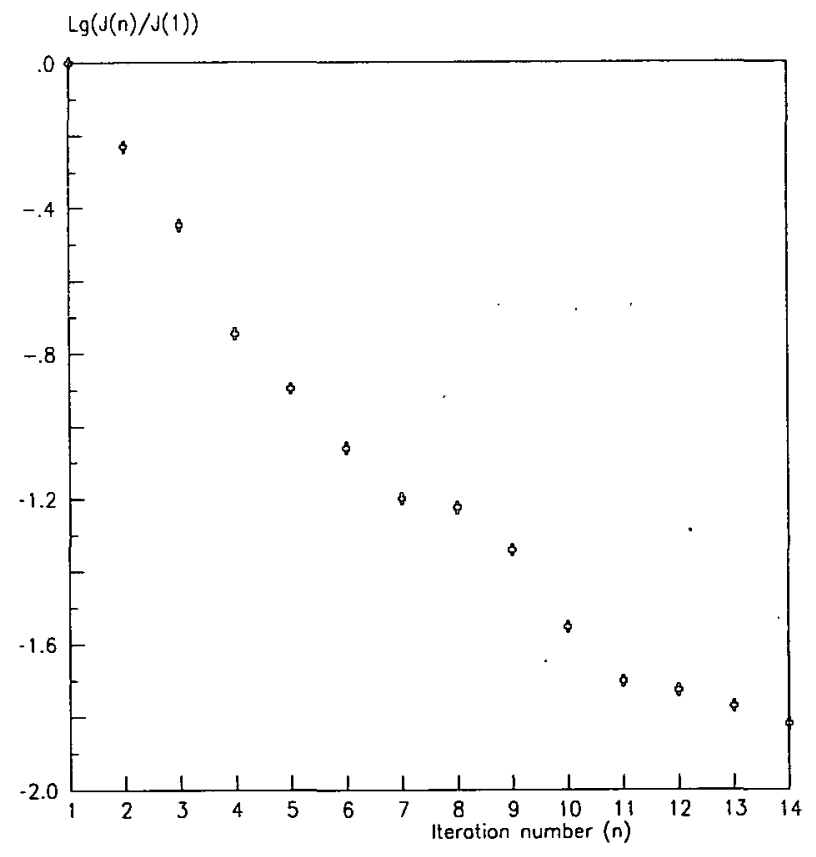

Figure 14: Objective function reduction versus iteration number. 